\title{
Más allá de una concepción instrumental del valor de los animales: la irracionalidad del paradigma humanista
}

\author{
(Beyond an instrumental conception \\ of the value of animals: the irrationality \\ of the humanist paradigm)
}

\author{
Olga CAmpos SERENA
}

Recibido: 28 de junio de 2011

Aceptado: 20 de diciembre de 2011

\section{Resumen}

En el artículo se lleva a cabo un análisis de las bases en las que descansa el paradigma ético tradicional. La pretensión es mostrar que la propuesta humanista falla en su pretensión de justificar que la responsabilidad moral de los agentes morales se dirige a todos y sólo a los seres humanos. En la parte final se apuesta por una propuesta alternativa más plausible.

Palabras clave: racionalidad moral, especieísmo, igualdad.

\begin{abstract}
The paper conducts an analysis of the foundations upon which rests the traditional ethical paradigm. The aim is to show that the humanist analysis fails when try to justify the moral responsibility of moral agents for all and only human beings. In the final part is defended a more plausible alternative proposal.
\end{abstract}

Keywords: moral rationality, speciesism, equality. 
El punto de partida de este trabajo es la necesidad de poder determinar qué papel han de jugar los animales no humanos en la evaluación moral de las situaciones en las que nuestros intereses se ven envueltos. La concepción de una comunidad moral en la que no tienen cabida los animales no humanos forma parte de las coordenadas éticas del paradigma humanista. La lectura cristiano-agustiniana de la concepción estoica y aristotélica llevó a interiorizar la idea de que los animales han sido creados con el único fin de satisfacer nuestras demandas ${ }^{1}$. Esto se traduce en una concepción instrumental de los mismos desde la que se admite que su consideración estará en función del servicio que presten a nuestros intereses².

Resulta entonces irrenunciable la necesidad de comprobar si las razones que ofrece la ética tradicional para negar la considerabilidad moral de los animales se sostienen. Y preguntar también si, al margen de sentimientos como el de compasión o benevolencia, podría defenderse en su caso la satisfacción de un criterio relevante para la pertenencia a la comunidad moral ${ }^{3}$.

A lo largo de la historia se han sucedido diversas revoluciones ideológicas que han tenido como consecuencia la ampliación progresiva del grupo de los miembros que forman dicha comunidad. Una visión histórica de los límites de la misma permite comprobar que el proceso tendría la forma de diferentes círculos concéntricos que cada vez abarcan a más individuos a los que se les reconocen derechos morales, y donde el siguiente paso sería reconocer el valor intrínseco de algunos animales. Ello no supondría un compromiso con una respuesta concreta a la pregunta por cuáles serían específicamente nuestras obligaciones hacia los animales, sino con la idea de que ellos también son la clase de individuos que tienen un valor en sí mismos.

Reivindico la necesidad de poner orden en la discusión, para la adecuada argumentación a favor del reconocimiento de dicho valor en los animales. Dado que la propuesta tradicionalmente asumida apuesta por la exclusividad humana en este sentido, se puede decir que el objetivo se cifrará en la pretensión de justificar la ampliación de la comunidad moral. Defenderé que la posición humanista no se sostiene en ninguna de las dos versiones en las que habitualmente se presenta.

\footnotetext{
${ }^{1}$ Es útil consultar al respecto García Trevijano (1999), donde accederemos a una selección textos filosóficos históricos importantes en la discusión por la consideración moral de los animales.

2 Hay que reconocer no obstante algunas excepciones históricas en este sentido. En 1892 Henry Salt publica Animal' rights, una exposición entonces novedosa del tema.

3 No puede pasarse por alto que la ampliación de la comunidad moral más allá de los seres humanos podría plantearse de manera más o menos restrictiva. Apostaré por la plausibilidad de un enfoque sensocentrista donde lo que cuenta para la atribución de valor intrínseco es la posibilidad de contar con un interés en evitar el dolor. Pero esta no es la única forma en que podría entenderse la crítica a la ética tradicional, pues existen también propuestas ecocéntricas y biocéntricas. Véase Schweitzer (1929), pp. 246-247 y Leopold (1949). Para conocer las dificultades tanto teóricas como prácticas de estos análisis holistas véase Schönfeld (1992), pp. 359-361 y Warren (1997), pp. 24-49.
} 


\section{La propuesta especieísta}

En un primer contexto humanista la especie a la que cada individuo pertenece se considera relevante. Lo que funciona como criterio de considerabilidad moral, de concesión de valor intrínseco, es la pertenencia a la especie Homo Sapiens. Por ello, desde este paradigma humanista, sólo los seres humanos tienen valor intrínseco. De tal forma que la pretensión no es encontrar un rasgo concreto común a todos los humanos, pues el mero hecho de ser humano ya concede en sí mismo una consideración moral especial.

Pero, en tanto que desde el punto de vista científico se considera el antropocentrismo como una posición anacrónica, parece lógico preguntar por qué tendría que seguir siendo el paradigma vigente en ética. Sin embargo, la idea en la que parece descansar esta propuesta es la de que no es necesario justificar por qué sólo nuestra especie tiene valor intrínseco. En este contexto que parece funcionar como una ideología, y al que DeGrazia (1996, pp. 56-57) se refiere como The sui generis view, se está produciendo una discriminación por el mero hecho de no pertenecer a una especie concreta. Ello suele conocerse como especieísmo y obedece a un fuerte prejuicio en torno a la exclusividad moral de los seres humanos (Midgley 1983, pp. 98-99)4.

El especieísmo es un tipo de discriminación tan insostenible como el racismo o el sexismo. Si se está interesado en afirmar que el sexo o la raza son características moralmente irrelevantes, entonces habrá que hacer lo mismo con respecto a la especie si se quiere ser coherente. La cuestión es que si se mantuviera con relación a la especie que las razones no cuentan, entonces el racista o el sexista podrían mantener lo mismo.

Si se quiere mantener que la analogía con el sexismo y el racismo no es apropiada, entonces deberá encontrarse algo que justifique por qué sólo los miembros de la especie Homo sapiens tienen valor intrínseco. Esto es, debería poder mostrarse la existencia de razones a favor de dicha exclusividad moral que llevaran a dejar de considerar arbitraria la propuesta. Por tanto, los interesados tendrían que identificar alguna característica responsable de nuestra superioridad en este sentido. Pero entonces habría que notar que en tal caso ya no estaríamos en un contexto propiamente especieísta, en tanto que ahora las razones contarían. Ello provocaría una discusión distinta a la que tiene lugar cuando se afirma que los únicos que merecen protección moral son los seres humanos por el hecho de serlo. Siendo esta última una afirmación arbitraria que descansa en un prejuicio y que, por tanto, no puede servir para justificar la concesión de valor intrínseco.

\footnotetext{
4 Hay quien ha apuntado la posibilidad de establecer una distinción con relación a esta idea también en el plano de la concreción de las obligaciones. Se trata de percatarnos de lo que M. L. Gerrek ha llamado especieísmo modificado. Lo que el autor pretende señalar es que actitudes tan diferentes por nuestra parte hacia especies distintas de animales no humanos (por ejemplo los domésticos y los destinados a la alimentación) resulta arbitrario e inconsistente. Véase Gerrek (2004).
} 
Además de la arbitrariedad, en otro plano podrían citarse algunas implicaciones prácticas que resultarían muy controvertidas. La discriminación a la que puede llevar una propuesta de este tipo queda de manifiesto con el planteamiento de una situación hipotética en la que nos encontrásemos con individuos idénticos a nosotros en todos los sentidos moralmente relevantes (capacidad de sufrir, de razonar, etc.) menos en la pertenencia a nuestra especie. Parece que estaríamos de acuerdo en que sería una consecuencia problemática el hecho de tener que dejar fuera del ámbito moral a tales individuos (LaFollette y Shanks 1996, p. 43).

En la misma línea señala D. DeGrazia (1996, pp. 58-61) que, asumiendo que el Homo erectus es la especie desde la que evolucionamos, resulta arbitrario mantener que si hubiera sobrevivido esta otra especie de homínidos sus miembros no merecerían estar dentro de la comunidad moral. No hay una separación genética clara entre ellos y nosotros, no es posible señalar el momento concreto en el que el Homo erectus mutó a Homo sapiens. Si analizamos este asunto desde el punto de vista de la información genética entonces no debemos dejar de tener en cuenta que, si bien los chimpancés son muy diferentes a nosotros en muchos aspectos, por otro lado somos enormemente parecidos genéticamente (compartimos un $98.77 \%$ del ADN). Si la biología usara un criterio basado en la relación genética entonces homínidos, chimpancés y quizás gorilas deberían formar parte del mismo grupo. Por tanto, además de la arbitrariedad, tampoco podríamos mantener de forma coherente que es la pertenencia a la especie Homo sapiens lo que tendría que determinar el que un individuo tenga o no valor intrínseco.

En la argumentación clásica en torno a la supuesta exclusividad moral de los humanos también suele introducirse en la discusión una referencia a la idea de persona como aquello que designa a los individuos con valor intrínseco. La idea que se asocia a esta versión del paradigma humanista es la de que ser una persona significa pertenecer a la comunidad moral y que en concreto los únicos individuos que forman parte de la misma son los seres humanos.

Resulta imprescindible profundizar en este concepto con el objetivo de evitar la ambigüedad que rodea al término y poder así determinar si se está introduciendo algún elemento relevante que pudiera modificar la evaluación del argumento humanista 5 . Hacerse cargo de la apuntada ambigüedad del término persona pasa por percatarse de la necesidad de distinguir un uso puramente normativo o evaluativo (moral o legal) y uno puramente descriptivo (uso convencional del término) (Feinberg 1980). Se habla de personas en un sentido normativo cuando se adscriben propiedades morales. De manera que atribuir personeidad de forma normativa a algún individuo supone atribuir cualidades morales como derechos o deberes pero no (necesariamente) algún tipo de característica observable (sea pertenecer a una

5 Si se quieren conocer los orígenes de la idea de persona y las definiciones clásicas del término que irán en esta línea puede consultarse DeGrazia (2006), pp. 40-42. 
especie particular o un determinado grado de conciencia). Sin embargo, un uso empírico o descriptivo del término sí nos exige que contemos con aquellas características que por convención harán de cualquier individuo que las posea una persona. De forma que utilizar el término en este sentido sí nos proporciona información acerca del individuo en cuestión. Si en este caso ser una persona depende de la posesión de las características a, b y c, entonces no debe haber problema en aceptar que alguien que las posea será persona en este sentido descriptivo. Es una cuestión de convención. Por ello Feinberg (1980) se refiere a ello como "the commonsense concept of personhood" (el concepto de personeidad del sentido común), e insiste en que este uso es meramente descriptivo y no supone la atribución de derechos, deberes o cualquier otra característica normativa.

Si bien estamos ante usos del término que podrían ser independientes, otra cosa es que pensemos que ese uso descriptivo puede ser útil para identificar las características que pueden servir como base para justificar la adscripción de derechos morales. De hecho hay quien podría estar interesado en admitir que debería haber una relación relevante entre ambos usos del término. De forma que, desde esta asunción, el grupo de individuos que se designa en cada uso debería coincidir. Los individuos con determinadas características acordadas que se consideran personas (uso descriptivo) serían los mismos que el grupo de individuos con valor intrínseco (uso evaluativo). Es decir, habría que evaluar la posibilidad de entender que aquellos individuos que habitualmente describimos como personas son los únicos que formarían la comunidad moral. Hechas las aclaraciones terminológicas, y suponiendo la relación propuesta entre los dos usos del término, vemos que el argumento humanista tendría en este contexto la siguiente forma:

1. Sólo las personas ${ }_{d}$ tienen valor intrínseco

2. Los animales no son personas ${ }_{d}$

3. Entonces, los animales no tienen valor intrínseco

Situados en el anterior contexto del paradigma humanista comprobamos un interés por que el criterio acordado para el uso descriptivo de persona sea la pertenencia a la especie Homo Sapiens. Lo que ello significa es que la personeidad sería una característica predicada exclusivamente de los seres humanos. De forma que el anterior argumento tendría que ser en este caso traducido como sigue:

1. Sólo los miembros de la especie Homo Sapiens tienen valor intrínseco

2. Los animales no son miembros de las especie Homo Sapiens

3. Entonces, los animales no tienen valor intrínseco

Desde la propuesta humanista entonces se estaría entendiendo que para ser persona, desde el uso descriptivo del término, hay que pertenecer a la especie Homo 
Sapiens (Sapontzis 1987, pp. 47-69). Así, puede verse que la idea de que la especificación evaluativa del término vendrá determinada por esta interpretación concreta del aspecto descriptivo del mismo nos conduce al mismo punto. La forma en la que aquí se está interpretando el criterio para la personeidad hace que el argumento acabe siendo igual; que se trate, en definitiva, de una propuesta arbitraria que nos permite catalogarla de especieísta.

Lejos del criterio de la especie es fácil comprobar que el uso descriptivo que habitualmente hacemos del concepto de persona se extiende a algunos casos más allá de la especie Homo sapiens 6 . Esto es, asociamos la personeidad a un determinado grupo de habilidades con independencia de la especie. Dejando al margen la cuestión de si es el hecho de ser una persona lo que debe asociarse al valor intrínseco, lo que ahora me interesa es comprobar que desde el momento en que dicho valor se relaciona con la presencia de determinadas capacidades nos situamos en un contexto muy distinto.

Si el objetivo dentro de la tradición humanista seguirá siendo el de perseguir la exclusividad moral de los seres humanos, ahora se comprende la necesidad de dar razones al respecto. Se mejora así la argumentación humanista analizada, proponiendo la racionalidad como criterio de concesión de valor intrínseco.

\section{La racionalidad moral y los humanos no racionales}

Es amplia la tradición filosófica en la que podría apoyarse la idea de que es la capacidad racional (el lógos de los griegos y la ratio de los latinos) lo que justifica la diferente consideración moral de humanos y animales. Sabemos que ya desde Aristóteles se define al ser humano como un animal racional. La racionalidad nos distingue de los animales no de un modo accidental sino esencial. Si lógos significa en griego a la vez razón y palabra entonces, tal como apunta Diego Gracia (2002, pp. 134-139), debemos situar aquí el origen del requisito de la capacidad de hablar como algo necesario para ser una entidad moral. Aristóteles reconoce que los animales tienen aisthesis, es decir, que son capaces de sentir placer y dolor. También pueden recordar, tienen mnéme. Por ambas cosas los animales tienen experiencia (empeiría). Ahora bien, aunque tengan las cualidades señaladas no son racionales, carecen de lógos, y siendo así no merecen consideración moral alguna (para los

\footnotetext{
${ }^{6}$ David DeGrazia señala el caso de Spock (de la serie Star Trek) y de los personajes del Planeta de los Simios como ejemplos en los que estaríamos dispuestos a catalogar como personas a individuos que no son humanos. Véase DeGrazia (2006), p. 41. Para el autor estos casos nos sirven para comprobar que la personeidad no depende de la especie sino de un determinado grado de conciencia que permita capacidades como autonomía, racionalidad, autoconciencia, competencia lingüística, sociabilidad, capacidad para llevar a cabo acciones intencionales y agencia moral. Véase DeGrazia (2006), pp. 44-46.
} 
griegos, la empeiría no necesita de lógos; por ello no es conocimiento sino algo previo). También en los textos bíblicos (Evangelio de Marcos 5, 1-13 y 11, 12-22) se recoge la herencia de la idea de superioridad del hombre 7 . La interpretación que se llevó a cabo desde el cristianismo de los presupuestos estoicos y aristotélicos pone el acento en una tajante separación entre humanos y animales en todos los planos. En el plano moral la consecuencia fue una concepción instrumental del valor de los animales que todavía perdura.

En la tradición racionalista occidental el hecho de que los humanos seamos los únicos capaces de entender las reglas del juego moral es lo que cuenta para restringir los límites de la comunidad moral. La capacidad de llevar a cabo juicios morales impersonales funciona aquí como condición de posibilidad para contar con protección moral. De manera que la capacidad de razonar moralmente es lo que se postula como relevante ${ }^{8}$. Por tanto, afirman, nuestro tratamiento de los animales no está gobernado por la ley moral.

Sin embargo, la mayoría de los representantes de esta postura insisten en que no podemos tratar a los animales de cualquier manera. Pretenden así hacerse cargo de nuestras intuiciones acerca de que debemos medir nuestra conducta con los animales en algún sentido. En tanto que no pertenecen a la comunidad moral, la intuición de que no debemos maltratarlos toma la forma de una obligación indirecta9 ${ }^{9}$ Por ejemplo, desde la doctrina kantiana se insiste en que no podemos tratar como nos plazca a los animales porque ello podría llevarnos a hacer lo mismo con los individuos autónomos. Así, las prescripciones indirectas no tendrán más fuerza que la de procurar evitar el endurecimiento de nuestro carácter moral10.

La protección moral que ello supondrá para los animales no será muy significativa. Además parece que la presunción de que la crueldad hacia los animales se extenderá a nuestras relaciones entre seres racionales es bastante gratuita. Tratar bien a los animales no es garantía de que se respeten los derechos de los seres humanos, ni tampoco al contrario. Habría que percatarse de que lo que realmente subyace tras esto es la intuición de que la capacidad de sufrir de los animales no es irrelevante. Por ello no se establecen obligaciones indirectas hacia los objetos y se niega que la máxima que permite maltratar a los animales pueda universalizarse.

\footnotetext{
7 Se puede encontrar un análisis del papel de los animales en la teología en Linzey (1994).

8 Podemos ver un amplio análisis de esta cuestión en Sapontzis (1987).

${ }^{9}$ Aunque suele asociarse el establecimiento de obligaciones morales indirectas hacia los animales con la doctrina Kantiana, no se debe olvidar que lo mismo ha sido defendido por otros pensadores históricos como Plutarco, santo Tomás o John Locke. De la misma forma se encuentran representantes actuales de esta idea, es el caso de Peter Carruthers. Véase Carruthers (1992). A nivel más general, Mary Midgley lleva a cabo un amplio análisis del papel de los animales en la tradición racionalista en su libro Animals and why they matter. Véase Midgley (1983).
}

10 Sobre el papel de los animales en la moral kantiana puede consultarse Cavalieri (2001), pp. 47-59. 
Algunos defensores de la atribución de derechos morales a los animales quieren resaltar lo que consideran un injustificado olvido de sus verdaderas capacidades cognitivas. Puede que algunos animales cuenten con un nivel de racionalidad suficiente que lleve a poder reconocer desde esta propuesta su valor intrínseco también. Los últimos avances en etología, neurofisiología y los nuevos diseños experimentales arrojan luz acerca de las capacidades reales de las demás especies. Unas capacidades muy superiores a las que hasta hace poco se les atribuía, y que ponen de manifiesto la necesidad de adoptar un enfoque gradualista al respecto. Lejos de las diferencias categóricas, en los últimos años se ha podido constatar la presencia de algunas acciones con un cierto grado de carga moral en determinados animales (DeGrazia 1996, pp. 208-210).

S. F. Sapontzis plantea que podríamos acordar mantener que en determinados contextos no sea necesario contar con una racionalidad de tipo humano para poder reconocer que se está llevando a cabo un acto moral. Propone distinguir dos dimensiones diferentes dentro del valor moral. Una que es independiente del agente y otra que sí estará en relación con el mismo. En el primer caso se refiere al valor moral de una acción que es independiente de la relación del agente con la acción. Por ejemplo cumplir una promesa o prevenir un asesinato son un tipo de actos a los que se les podría atribuir un determinado valor moral al margen de las razones del agente para llevarlos a cabo o de si el agente es consciente o no de que está realizando tales actos. En el segundo caso se refiere a la existencia de una dimensión distinta del valor moral que sí depende de la comprensión por parte del agente de la situación y de su acción, incluyendo su significado moral y sus motivos para actuar ${ }^{11}$. La cuestión es que es sólo la segunda de las dimensiones la que se refleja en el argumento clásico. Reconocer también la existencia de una dimensión del valor moral independiente del agente podría permitirnos contemplar a algunos animales como agentes morales en un determinado grado.

Sin embargo, puede que los defensores del paradigma racionalista insistan en que la capacidad que ellos están proponiendo sí requiere poder actuar conforme a razones; unas razones que serían compartidas por cualquier otro individuo situado en el mismo lugar. Y se requeriría que uno sea capaz de respetar los derechos morales de los demás. En definitiva, una racionalidad moral que demanda un determinado tipo de complejidad moral del que hemos de admitir que carecen los animales.

Ahora bien, si es esta última la interpretación correcta del requisito racionalista, entonces además de los animales habrá otro grupo de individuos incapaces de satisfacerlo. Me refiero al conjunto de seres humanos que tienen unas capacidades cognitivas por debajo de las que poseen los seres humanos adultos normales. Bebés,

${ }^{11}$ En palabras del autor: «while an honest act done to impress a friend has equal moral ${ }_{a i}$ value with an honest act done because it is honest, the moral ${ }_{\mathrm{ad}}$ value of the second action is superior to the moral $\mathrm{ad}_{\mathrm{ad}}$ value of the first action. Finally, "moral" can be used in the ordinary, inclusive sense to refer to the moral $_{\text {ai }}$ plus moral ${ }_{\text {ad }}$ value of an action». Sapontzis (1987), p. 31. 
niños pequeños e individuos con una discapacidad cognitiva grave (conocidos en su conjunto habitualmente como casos marginales humanos) presentarán la misma dificultad que los animales para la racionalidad moral.

Desde el argumento de los casos marginales se quiere poner de manifiesto una incoherencia en el planteamiento de aquellos que defienden la capacidad de razonar moralmente como criterio para atribuir valor intrínseco12. La cuestión es que si es este el requisito entonces tanto los animales como los casos humanos señalados carecerían de tal valor. Pero el hecho es que aquellos que proponen este criterio a su vez insisten en que tal grupo concreto de humanos sí tienen valor intrínseco. De forma que no se podría mantener, con coherencia, que el hecho de no contar con la apuntada capacidad hace que los animales carezcan del pertinente valor. Hasta ahora no sabemos cuál sería el criterio de considerabilidad moral más adecuado, y por tanto tampoco sabemos qué individuos tendrán valor intrínseco, pero lo que sí es fácil ver es que el esquema racionalista es incoherente. El argumento podría esquematizarse de la siguiente forma ${ }^{13}$ :

1. Si una capacidad tal como la agencia moral es necesaria para la considerabilidad moral entonces los animales no son moralmente considerables.

2. Los humanos marginales son moralmente considerables a pesar del hecho de que ellos carecen de agencia moral.

3. Si los humanos marginales son moralmente considerables, entonces ninguna capacidad de la que ellos carecen puede ser necesaria. (Tanner 2006, p. 52)

Lo que parece haber debajo del punto de vista tradicional es la pretensión de mostrar que todos los humanos son moralmente considerables pero no los animales. Algo que pasa por poder encontrar alguna capacidad que posean todos los humanos y de la que a su vez carezcan los demás animales. Pero la característica propuesta, la racionalidad moral, no les permite alcanzar justificadamente su objetivo.

Su pretensión de combinar el criterio racionalista (que aseguraría la irrelevancia moral de los animales) con la atribución de valor intrínseco a los humanos marginales les lleva a explorar distintas posibilidades que sirvieran para explicar por qué dicho grupo de humanos forman parte de la comunidad moral aunque no sean capaces de racionalidad moral.

\footnotetext{
12 La primera referencia se encuentra en Jan Narveson (1979), p. 164. Otros autores reivindican la terminología argument from species overlap por entender denigrante la más usual. Véase al respecto Dombrowski (1997) y Wilson (2005).

13 Ha habido muy diversas formulaciones del argumento de los casos marginales. Si bien insisten en el mismo punto la presentación del argumento puede variar. Julia Tanner recoge distintas versiones de diferentes autores como Peter Singer, Richard Ryder o Andrew Linzey. Para ver citados algunos fragmentos relevantes al respecto y las referencias concretas véase Tanner (2006), p. 51. Es especialmente interesante ver cómo D. A. Dombrowski analiza en profundidad el argumento de los casos marginales en su volumen Babies and Beasts.
} 
Los defensores del paradigma racionalista quieren rebatir la acusación de incoherencia afirmando que el criterio propuesto sí es lo que determina el valor intrínseco, pero mantienen que la clave está en percatarnos de que los humanos marginales son un caso especial porque hay importantes razones en juego para que así sea. Sin duda el objetivo ya es cuestionable desde el principio, al margen del resultado de la evaluación de las apuntadas razones. No obstante podría preguntarse acerca de qué podría ser eso que hace a los humanos no racionales tan especiales en la evaluación, a diferencia de los animales no humanos.

Desde el conocido argumento de la pendiente resbaladiza se mantiene que conceder valor intrínseco sólo a los humanos que posean racionalidad moral podría desencadenar un peligroso abuso, esto es, podríamos caer en una pendiente resbaladiza que nos llevaría finalmente a justificar toda clase de atrocidades morales. Este argumento, mantienen, serviría para justificar la inclusión de los humanos marginales en el ámbito moral y dejar fuera a los animales, puesto que lo último no tendría la consecuencia señalada (Carruthers 1992, pp. 115-120). Pero la cuestión es si apelar a la posibilidad de caer en una pendiente resbaladiza podría funcionar como justificación. Parece que dudosamente. Sabemos que en muchas sociedades humanas tradicionales no estaban de acuerdo con la idea de que todos los humanos contaran con protección moral, y sin embargo esto no les llevó a caer en la señalada pendiente ${ }^{14}$. Es cierto que en muchos casos se llevaban a cabo determinadas prácticas, la del infanticidio por ejemplo, como la única forma de sobrevivir en un medio hostil. No obstante también son comunes otras sociedades en las que la exclusión de algunos humanos no obedecía a cuestiones relacionadas con la supervivencia, sin que ello desencadenara las consecuencias que predice el argumento. Por ejemplo, en la Roma y Grecia clásicas fue habitual la práctica del infanticidio en este sentido. También en el Japón del siglo XVIII y entre algunos grupos de la Polinesia 15.

No obstante, al margen de estas razones es obligado preguntar si la forma en que el argumento otorga derechos a los humanos marginales acaso no sería demasiado indirecta. Su valor intrínseco estaría dependiendo de la anticipación de posibles consecuencias que consideramos indeseables desde un punto de vista moral. Parece claro que, si la pretensión es justificar por qué los humanos incapaces de racionalidad moral merecen estar dentro de la comunidad moral, lo más adecuado sería encontrar un criterio de inclusión relevante que éstos satisfagan.

Por otro lado, también se ha apelado a la idea de equilibrio reflexivo rawlsiano señalando que debemos partir de las creencias morales comunes para, desde ahí, construir una teoría moral plausible. Tales creencias morales comunes, afirman, pasan por incluir a todos y sólo los seres humanos en el ámbito moral, esto es, esta-

14 Un análisis más extenso puede encontrase en Dombrowski (1997), pp. 128-129.

15 Si interesa indagar en esta cuestión véase Kuhse y Singer (1985), pp. 99-107. 
ríamos ante una razón para incluir en la comunidad moral a los casos humanos marginales aunque no satisfagan el criterio de la racionalidad moral ${ }^{16}$. Peter Carruthers (1992, pp. 16-111) entiende que desde un marco teórico como éste (esto es, asumiendo la racionalidad moral como criterio inclusivo y el punto de vista del equilibrio reflexivo) estaremos en disposición de poder explicar por qué tales humanos deben estar dentro de la comunidad moral sin que ello suponga tener que hacer lo mismo en el caso de los animales. Pero si asumiéramos la idea de equilibrio reflexivo no deberíamos pasar por alto que éste también nos exige coherencia en nuestros planteamientos. Lo que ello implica es que deberíamos tratar casos semejantes de forma semejante, pues en la moral no puede haber dobles baremos. Si no encontramos ninguna diferencia moralmente relevante entre ambos grupos entonces tendremos que admitir que estamos ante casos semejantes (individuos incapaces de racionalidad moral). Está en juego nuestro concepto de justicia. La necesidad de tratar de forma similar casos similares es una importante creencia moral común que nos llevaría a cuestionar el objetivo inicial de los humanistas (Dombrowski 1997, pp. 124-127).

Para muchos la apuntada similitud acaba al percatarnos de la potencialidad de los humanos marginales para llegar a ser individuos que pueden satisfacer el criterio inclusivo. La potencialidad para llegar a tener las capacidades de los adultos normales, y así poder contar entonces con racionalidad moral, sería lo que justifica que ellos tengan valor intrínseco mientras que los animales no (Leahy 1991, pp. 18-25). Pero es fácil percatarse de que, independientemente de la relevancia del argumento, éste no es capaz de abarcar a todo el grupo de los humanos incapaces de racionalidad moral, como es el caso de los humanos cognitivamente discapacitados. De forma que este análisis, si algo pudiera mostrar, será sólo con respecto a los niños pequeños (Dombrowski 1997, pp. 122-124) ${ }^{17}$. Pero la cuestión fundamental es que resulta dudoso que lo que se plantea desde el argumento pueda funcionar a modo de justificación. La potencialidad de un niño pequeño para llegar a ser un adulto (con las capacidades que le permitiría satisfacer el criterio) no nos legitima para tratarlo

$16 \mathrm{~J}$. Rawls entiende que en el caso de las obligaciones morales es necesario tener un sentido de la justicia, puesto que éstas se adquieren mediante el acuerdo. Pero sabiendo que tenemos obligaciones de justicia hacia los humanos no autónomos, sería obligado preguntar por qué el velo de ignorancia en la posición original no es lo suficientemente opaco como para que desconozcamos también la especie a la que perteneceremos. La mayoría de los contractualistas responden afirmando que el pacto sólo tiene sentido entre individuos racionales. Se hace una excepción con los humanos no racionales porque ello es lo que parece exigirse si perseguimos un equilibro reflexivo. Véase Rawls (1971), pp. 505-512.

17 Tampoco puede pasarse por alto que asumir el argumento de la potencialidad nos comprometería con una postura abolicionista en torno al aborto y la investigación con embriones. Si algunos defensores de este argumento intentaran desligarse de la idea de que es malo permitir el aborto por elección o la investigación con embriones no podrían porque tendrían muchos problemas para explicar por qué los niños pequeños tienen valor intrínseco debido a su potencialidad y sin embargo los fetos humanos o los embriones, que también la tienen, carecen de tal valor. Consúltese Regan (1999), pp. 21-29. 
como tal desde el principio. El que alguien tenga la capacidad de llegar a ser X no es razón suficiente para atribuirle los derechos de X, pues no tiene las características que le hacen ser a $\mathrm{X}$ portador de esos derechos.

También suele plantearse el argumento de las clases, desde el que se sugiere que todos los humanos, con independencia de sus capacidades individuales, poseerán valor intrínseco porque los humanos son la clase de individuo que posee dicho valor moral. Si el argumento fuese correcto entonces los humanos marginales pertenecerían a la comunidad moral mientras que los animales no. A pesar de que podríamos reconocer diferentes versiones de este argumento todas ellas insisten esencialmente en el mismo punto18: es la clase a la que uno pertenece, y no las capacidades individuales, lo que importa a la hora de determinar la considerabilidad moral. Entonces, según el argumento, un humano marginal tendrá estatus moral porque al ser miembro de una clase determinada, la nuestra, está en relación con aquellos (los humanos normales) que tienen valor intrínseco. Pero resulta difícil compatibilizar nuestras intuiciones en torno a la protección moral que entendemos que merecen con el hecho de que éstos acaben recibiendo su estatus moral por asociación. Además, al margen de ello, el problema es que muchas veces no se sabe exactamente a qué se refieren los proponentes del argumento cuando hablan de clase. Para Tanner (2006, p. 54), interesada en cuestionar el argumento, podría entenderse "ser de una clase" como "tener las características normales del grupo". Pero hacer descansar el estatus moral en cómo son la mayoría de los humanos sería contingente. Si la mayoría de los humanos perdiera su nivel de racionalidad entonces los animales no humanos sí tendrían estatus moral. Una conclusión que los proponentes del argumento no estarían dispuestos a aceptar. También podría interpretarse "ser de una clase" como "tener las características naturales del grupo". Pero surgiría un problema en torno a la veracidad de la conclusión: el hecho de que la mayoría de los humanos sea naturalmente racional no significa que todos los humanos lo sean. Además, una cuestión añadida es que resulta problemática la idea de que hemos de concebir las especies como clases naturales. La teoría de la evolución de Darwin apunta en este sentido: las especies no son inmutables, en tanto que hay individuos que evolucionan hasta formar nuevas especies (trasmiten los rasgos nuevos a la siguiente generación). Si esto es así no parece que tenga mucho sentido hacer descansar la atribución de valor moral en la idea de "clase natural". La pretensión de que ello coincida con los límites de nuestra especie tal como los concebimos hoy es una posición anacrónica y obvia la contingencia. Pero las críticas a las que se ve expuesto el argumento de las clases no acaban aquí, puesto que desde el mismo tam-

\footnotetext{
18 Son muchos los autores que de una u otra forma han mantenido esta idea. Thomas Scanlon, Carl Cohen o Roger Scruton son algunos de ellos. Julia Tanner repasa el núcleo de sus propuestas al respecto. Se pueden encontrar las referencias concretas en Tanner (2006), pp. 53-54.
} 
bién se podrían elaborar argumentaciones racistas y sexistas ${ }^{19}$. Como afirma Peter Singer (1974, pp. 124-126), si dado el estado mental de los humanos marginales reconocemos injusta la idea de aprovecharnos de un defecto aislado, entonces no está claro qué podría servir para justificar el aprovecharnos de una limitación más general. Es decir, entendemos razonable que el hecho de que tales humanos no alcancen la complejidad mental de los demás no es una buena razón para negarles consideración moral. Si esto es lo que argumentamos para estos casos particulares de humanos lo mismo debería ser mantenido en el caso de otros grupos que, aunque más amplios, se encuentran en una situación cognitiva paralela. Parece que tenemos que reconocer que la argumentación es especieísta, en tanto que al final la forma en que aquí se intenta justificar el valor intrínseco de los individuos humanos que no cumplen el criterio acaba descansando en el hecho de que son miembros de nuestra propia especie.

Tampoco resulta adecuado hacer referencia al supuesto vínculo que nos une a todos los humanos como aquello que podría ayudar a determinar el grupo de los que han de contar con valor intrínseco aunque no sean capaces de razonar moralmente. Se estaría apelando a la especial relación que supuestamente establecemos entre todos los humanos y al vínculo que ello genera entre nosotros (Nelson 1985, pp. 1520). Ahora bien, lo que puede estar criticándose desde aquí es el igualitarismo universal de algunas posiciones éticas para pasar a mantenerse que si podemos dar prioridad a los intereses de la familia y amigos entonces estará legitimado hacer lo mismo con los miembros de nuestra especie 20 . Pero el hecho es que apuntar a la posibilidad de reconocer las afecciones parciales en la determinación de las obligaciones morales no significa tener que negar valor intrínseco a aquellos hacia los que no tengamos tales afecciones (Sapontzis 1987, pp. 139-140; 151-154)21. Además, la exigencia de imparcialidad que caracteriza a la ética se vería desafiada si se admitiera la posibilidad de realizar juicios morales fundamentados en exclusiva desde posiciones parciales.

Los diferentes argumentos analizados quieren explicar por qué tendríamos también que conceder valor intrínseco a los humanos sin racionalidad moral a pesar de no satisfacer el criterio propuesto en segunda instancia desde el paradigma humanista. Se ha apuntado dónde se sitúan las dificultades en cada caso particular y es fácil ver que pesa como idea de fondo los beneficios que obtenemos del hecho de dejar fuera de la esfera moral a los animales no humanos. Las diferentes estrategias

19 Puede verse un tratamiento más sistemático de estas objeciones en Tanner (2006), pp. 54-59. Además es muy interesante el análisis que lleva a cabo S. D. Wilson en su artículo "The species-norm account of moral status". Véase Wilson (2005).

20 Puede verse el interesante análisis que al respecto lleva a cabo Jeff McMahan partiendo de la propuesta de T. M. Scanlon. Véase McMahan (2002), pp. 217-228 y Scanlon (1998), p. 185

${ }^{21}$ Debería reconocerse la diferencia entre la pregunta de por qué ciertos individuos tienen valor intrínseco y la de cuánto peso tienen los intereses de cada individuo. 
analizadas introducen una adición $a d$ hoc. La clausula ad hoc es que no sólo quienes tienen racionalidad moral, sino también aquellos que son similares, pertenecen a la misma clase, son potencialmente racionales, etc. tienen valor intrínseco. El procedimiento no es aceptable si se añade una nueva clausula sin añadir nueva información sobre lo que realmente cuenta para la concesión de valor intrínseco.

Los defensores del paradigma humanista en su formulación mejorada no han sido capaces de encontrar alguna característica moralmente relevante que distinga a esos casos humanos marginales de los animales no humanos. Pero lo fundamental es lo cuestionable de la pretensión de concederles a todos los humanos valor intrínseco aunque haya algunos que no puedan cumplir el criterio de considerabilidad moral propuesto. Se presenta con fuerza el hecho de que en ambos casos hay un paralelismo cognitivo que no puede pasarse por alto a la hora de concretar las responsabilidades morales de los agentes morales. Si se optara por mantener como criterio inclusivo la racionalidad moral, la única forma de evitar la acusación de incoherencia sería excluir de la esfera moral tanto a los animales como a los individuos humanos que no satisfacen el criterio. O bien podría optarse por un criterio distinto, la capacidad de sentir, que permitiría asegurar directamente el valor intrínseco de los humanos marginales y nos llevaría a mantener lo mismo con respecto a muchos animales.

\section{La igualdad como ideal moral: la plausibilidad de la alternativa sensocentrista}

Aunque tradicionalmente sólo se contempla la posibilidad de establecer obligaciones indirectas hacia los animales, la propuesta que reivindica la ampliación de la comunidad moral parece más plausible que su alternativa. Permitiría evitar un paradigma alternativo (el humanista) que nos hace ser especieístas, o incoherentes, o nos lleva a otorgar sólo un valor instrumental a los humanos no racionales.

Si compartimos la pretensión de que todos los miembros de nuestra propia especie formen parte de la comunidad moral entonces no se puede proponer ninguna característica relacionada con la racionalidad moral como criterio de considerabilidad moral si se quiere ser coherente. Es fácil ver que la inteligencia, la autonomía o la agencia moral no son compartidas por todos los seres humanos, y que postulamos valor intrínseco al margen de las diferencias que tienen lugar al respecto de tales características relacionadas con la racionalidad moral.

Es habitual apelar al importante grado de similitud que existe entre todos los seres humanos como una razón que permite atender a las demandas de liberación de los diferentes grupos humanos oprimidos históricamente (esclavos, mujeres, etc.) sin que ello nos llevara a tener que reconocer las exigencias morales de quienes postulan la liberación animal. Su razonamiento apunta a que las similitudes de todos 
los humanos nos llevarían a prescribir iguales derechos para todos nosotros, insistiendo en la relevancia moral de lo que supone que ése no pueda ser el caso de los demás animales. La idea sería que las diferencias entre ellos y nosotros son grandes, lo que imposibilita compartir derechos. Sin embargo es fácil reconocer también la existencia de diferencias relevantes dentro de nuestro propio grupo que llevaría a la necesidad de postular derechos diferentes (por ejemplo, hablar del derecho al aborto es algo que sólo tiene sentido en el caso de las mujeres). Lo que ello sugiere es que el hecho de que existan diferencias no es un obstáculo para que el principio básico de la igualdad sea extensivo a todos los miembros de la especia humana. Ahora bien, la única manera de que ello sea posible es asumiendo que el principio de igualdad no descansa en características de tipo fáctico, pues ello haría imposible la pretensión.

Abandonar la búsqueda de una igualdad real es condición de posibilidad para una efectiva aplicación del principio de igualdad a seres humanos muy diferentes. La clave está en percatarse de que el principio descansa realmente en un ideal moral: debemos tratar a los demás de manera que sus intereses iguales cuenten de la misma forma. Esta idea permite lograr una adecuada justificación del principio, a la vez que es compatible con el reconocimiento de diferentes derechos en función de la existencia de diferentes intereses (Singer 1974, pp. 109-112).

Entender la igualdad como igual consideración nos permite justificar la pretensión de que el principio se extienda a todos los seres humanos, pero también nos lleva a tener que reconocer que ha de ser también legítimamente aplicado a muchos animales no humanos. Estos tendrán intereses que habrán de ser igualmente considerados. El interés más básico es el interés en no sufrir, una característica que comparten todos los individuos capaces de tener experiencias conscientes negativas. Dado que el objetivo primordial de la moral es evitar que los individuos se vean dañados, la pretensión de prestar consideración al señalado interés se ve respaldada desde lo que podría concebirse como un imperativo.

La idea que subyace es la de que los propios intereses son, desde un punto de vista neutral, iguales en importancia a los intereses de cualquier otro. Este principio de igual consideración de intereses está en línea con la forma en que autores como Bentham o Sidgwick incorporaron esta idea de igualdad dentro del contexto de una ética utilitarista. Una idea que puede formularse de formas diferentes pero cuyo núcleo esencial parece un lugar común también de la filosofía moral más contemporánea.

El principio de igual consideración de intereses ha sido objeto de un amplio análisis, dentro del cual también encontramos apuntados los aspectos en los que éste puede resultar más problemático. Surge la discusión en torno a cuestiones de tipo metodológico, como la pregunta en torno a la posibilidad de lograr identificar las situaciones en las que estamos ante intereses iguales (DeGrazia 1996, pp. 11-35, 72- 
74). Sin embargo parece que habría que aceptar que una comparativa a grandes rasgos es plausible pues, aunque se reconozca que no puede compararse con exactitud entre seres humanos diferentes ni entre especies distintas, muchas veces la exactitud no es necesaria. En la mayoría de las ocasiones podremos identificar con facilidad dónde se está dando el sufrimiento mayor sin necesidad de comparaciones exactas (Singer 1979, pp. 76-77).

Hay autores que han preguntado si realmente es algo tan obvio que la razón requiere la adopción del principio de igual consideración de intereses (Maclean 1993, pp. 55-60). La idea de que la racionalidad requiere imparcialidad, y que la imparcialidad requiere la adopción del principio de igualdad podría ser problemática. Un juicio, mantiene Maclean, puede ser imparcial o desinteresado y a la vez incompatible con la idea de que debemos dar igual peso a los intereses de todos aquellos afectados por nuestras acciones. La conexión no es necesaria porque este elemento imparcial definitivamente puede estar presente en la justificación de una acción que sea incompatible con el hecho de otorgar la misma consideración a los intereses de todos. Lo que habría que analizar es si esto implica que se podría llegar a justificar que nuestros intereses pesan más que los de los animales no huma$\operatorname{nos}^{22}$. Podrían instarnos a comprender que estaría en juego un tipo de razonamiento similar al que nos permite justificar la legitimidad de optar por salvar la vida de nuestro propio hijo frente a la de un desconocido. Sin embargo habría que preguntar si realmente podemos fundamentar la existencia de un vínculo exclusivo entre todos los seres humanos al estilo del que un padre tiene hacia su hijo. Algo más que dudoso que por otro lado requeriría mostrar la pertinencia de que tal característica juegue dicho papel en la evaluación moral. Porque por un lado, además de que la relación paterno-filiar presenta características únicas, hay quienes desarrollan también vínculos fuertes hacia algunos animales, y por otro el requisito de imparcialidad propio de la ética parece quebrarse si se admite el grado de parcialidad demandado por la argumentación. No deja de parecer intuitiva, por una cuestión consistencia lógica, la idea de que dos intereses iguales en no sufrir merecen la misma consideración con independencia de quiénes los tengan.

Afirmar que los intereses de todos los humanos pesan lo mismo mientras que los de los individuos que pertenecen a otras especies pesan menos no es fácil de justificar. Podría entenderse que se falla en términos de consistencia lógica porque en realidad lo que se está proponiendo es una moral para los humanos y otra distinta para los animales, sin poder explicar cómo se relacionan o por qué son diferentes.

\footnotetext{
22 Bonnie Steinbock también ha querido desafiar el análisis que hace Singer del principio de igualdad, al entender que los resultados a los que la interpretación singeriana nos lleva resultan demasiado contraintuitivos. Frente a ello la autora reivindica una posición privilegiada para nosotros en la comunidad moral. Puede profundizarse en su propuesta consultando Steinbock (1978), pp. 247-256.
} 
Parece que hemos de considerar igual los intereses iguales de todos los que los tengan sin importar la especie, lo que supone formular la regla formal de justicia ${ }^{23}$.

Los aspectos esenciales que deberíamos tener en cuenta sobre el mencionado principio de igual consideración de intereses serían los siguientes. En primer lugar, estamos ante un principio formal, lo que significa que se dirige a la forma que debe tener el razonamiento moral y no al contenido. Lo único que nos está diciendo es que debemos tratar de igual forma los casos iguales. En segundo lugar, el principio de igual consideración no hace que necesariamente tengamos que tratar a todos los individuos como iguales en todos los sentidos (diferentes intereses darán lugar a un trato distinto). En tercer lugar, este principio es un componente necesario de cualquier teoría moral (Francione 2000, pp. 82-85).

Desde el paradigma humanista no se propuso una característica equivocada, más bien se trata de ver que es innegociable que hemos de partir de lo que podría entenderse como una condición de posibilidad de la nueva interpretación del principio de igualdad. Querer atender por igual a los intereses de los individuos nos obliga a contar con el prerrequisito que posibilita la presencia de tales intereses, a saber, la capacidad para experimentar sufrimiento y felicidad. La igual consideración refiere a los intereses individuales y éstos sólo estarán presentes en el caso de que uno pueda verse afectado por las acciones de los demás, lo cual vendrá determinado a su vez por la capacidad para sufrir. No hay justificación moral para no tomar en cuenta el sufrimiento de cualquiera que pueda experimentarlo. Entender la idea de igualdad como un principio de igual consideración nos permite estar en línea con esta idea tan básica.

No es difícil ver que la sensibilidad es la característica moralmente relevante si tenemos presentes los requisitos que debería satisfacer un criterio inclusivo para que su asunción estuviera justificada. Tiene que ser general, esto es, aplicable a cualquier entidad y capaz de resolver todas o la mayoría de cuestiones en torno al estatus moral. Debe conectar el estatus moral a propiedades empíricas del individuo. Por supuesto tiene que ser moralmente relevante. Y dando un paso más habría que añadir un cuarto prerrequisito tomado de James Rachels (1990). El autor indaga en las razones que justificarían una diferencia de trato y resalta la necesidad de tener en cuenta que lo que puede justificar un tratamiento diferente dependerá de la clase de trato que esté en cuestión en cada caso 24 . Lo que esto implica es que debe haber una conexión entre el estatus propuesto y el criterio adoptado. En el análisis

\footnotetext{
${ }^{23}$ De una forma u otra suele mantenerse que el principio de igualdad es básico en ética. Podemos ver un análisis de cómo se articula este principio en teorías éticas muy diversas consultando DeGrazia (1996), pp. 45-46.

24 Por ejemplo, a la hora de justificar por qué administramos la única dosis de penicilina disponible al enfermo A o B parece que lo relevante es el tipo de dolencia de cada uno y no sus expedientes académicos. Véase Rachels (1990), p. 176.
} 
de Cavalieri (2001, p. 32) ello se traduce en que el criterio inclusivo debe poseer un tipo de relevancia que podríamos definir como contextual. Es obvio que el nuevo criterio cumple con esta exigencia de relevancia contextual mejor que las propuestas de corte racionalista. La capacidad de sentir se relaciona con un interés en evitar el sufrimiento y parece que ello, y no un alto grado de racionalidad, es lo relevante si el contexto es el de la concesión de protección moral.

Puede justificarse la asunción del criterio de la sensibilidad desde el imperativo de no dañar. Es así porque los individuos conscientes que no son racionales sin duda pueden verse dañados con las acciones de los que sí lo son, y esto es algo que debería poder ser tenido en cuenta en la determinación de nuestra responsabilidad moral. En tanto que desde la moral hay una preocupación por proteger a los individuos del daño, lo lógico es hacer efectiva tal protección para todo el que tenga un interés en evitar el sufrimiento. Si los seres conscientes irracionales cuentan con el apuntado interés, entonces parece justificado abandonar como criterio de considerabilidad moral la racionalidad moral y defender en su lugar la capacidad de sentir.

La propuesta de la racionalidad moral como criterio inclusivo daba lugar a una distinción entre agentes y pacientes morales en la que sólo los primeros formaban parte de la comunidad moral. Pero deberíamos preguntarnos por la coherencia de defender que los agentes morales, encargados de determinar las obligaciones morales, son individuos más valiosos (Warren 1997, pp. 121-164 y 224-225). Porque resulta bastante difícil admitir que las personas con la capacidad en cuestión que deciden ser inmorales sean más valiosas que otros individuos que carecen de ella. Es más, si asumiéramos el argumento, no habría una razón plausible para no creer que la habilidad para elegir ser inmoral quita valor al grupo de los que tienen autonomía moral de la misma forma que se mantiene que la habilidad para elegir ser moral lo añade (Gruzalski 2004, pp. 3-11).

Finalmente resulta arbitrario entender que sólo los que puedan llevar a cabo actos morales tendrán valor intrínseco (Sapontzis 1987, pp. 1-46). Por ello habría que superar una concepción estrecha del valor y dejar de ver extensiblemente equivalentes las preguntas: ¿quién formula los principios? y ¿para quién se formulan los principios? La posibilidad de diferenciar tales preguntas pasa por defender una fundamentación de la ética no estrictamente racionalista.

Por tanto, una cosa es que los agentes morales sean los encargados de determinar las obligaciones morales y otra distinta que ellos sean los únicos beneficiarios de pleno derecho de tales obligaciones. La idea de que puesto que los seres humanos son los únicos que reconocen deberes también deben ser ellos los únicos beneficiarios de derechos puede ser cuestionada si reconocemos como nuevo criterio de considerabilidad moral la sensibilidad. Ello servirá para darle un nuevo sentido a la distinción clásica entre ser agente y ser paciente moral. Los agentes morales son aquellos individuos con responsabilidades morales mientras que los pacientes 
morales son aquellos que pueden resultar beneficiados o perjudicados según se determinen tales responsabilidades 25 .

Atendamos por un momento a varias intuiciones presentes en el fondo de esta discusión. Parece que estamos interesados en poder reconocer una adecuada protección moral a los humanos marginales, lo cual pasa por poder asegurar su valor intrínseco. Al mismo tiempo en general estamos dispuestos a reconocer una importante diferencia en el plano moral entre niños pequeños o discapacitados cognitivos incapaces de racionalidad moral y aquellos casos que son objeto de una toma de decisiones en contextos biomédicos, como es el caso de los humanos en estado vegetativo persistente y de los embriones (Dombrowski 1997, pp. 25-40). Por otro lado, también debemos percatarnos de que la intuición en contra del maltrato gratuito de los animales no se agota en el reconocimiento de deberes indirectos.

Habría que ver en qué descansa el interés por proteger a tan diversos individuos mientras que no a otros. Parece que en la asunción del imperativo de no dañar, sabiendo que la capacidad de sentir es lo que se relaciona con la experiencia de sufrimiento y que el sufrimiento es condición de posibilidad del daño. De forma que pasar a defender la apuntada capacidad como criterio inclusivo no es arbitrario puesto que no estamos ante una propiedad biológica más. Estamos ante algo definitivamente ligado al ámbito moral en tanto que la moral parece surgir de nuestra preocupación por determinados daños que parecen injustos ${ }^{26}$.

Sin duda el éxito del paradigma animalista está sujeto a la constatación de experiencias mentales negativas en los animales no humanos. El punto de partida para atribuir a muchos animales las emociones conscientes que posibilitan su capacidad de sentir se sitúa en el abandono de la tesis del abismo ontológico y su sustitución por la del continuo evolutivo con gradaciones, donde se niega la existencia de una discontinuidad radical entre el mundo de los animales humanos y no humanos. Tendrá un papel determinante el argumento de la analogía, que viene dado por el conjunto de varias argumentaciones que ponen el acento en las similitudes de

\footnotetext{
25 En palabras de Pablo de Lora: «[...] sólo algunos seres humanos alcanzan la condición de agente moral. Pero esto sólo nos permite identificar a los protagonistas de la ética, no necesariamente a su objeto, al ámbito de aplicación de las restricciones a nuestro comportamiento en la forma de ciertos deberes morales. Podría resultar que, entre los destinatarios de tales obligaciones, hubiera que incluir no sólo a quienes son agentes morales sino también a los pacientes morales, es decir, a aquellos que se ven afectados por las acciones de los demás sin ser ellos mismos agentes». De Lora (2003), p. 135. 26 Como ha afirmado Francisco Lara: «La idea no parece tan descabellada. Si se acepta normalmente que el objetivo esencial de la moralidad es fijar normas de conducta que prohíban dañar al otro, parecería arbitrario inferir que sólo los seres racionales pueden ser objeto de nuestras obligaciones morales. ¿Es que no pueden ser dañados los irracionales? Al fin y al cabo se trata de seres cuyo sufrimiento no se reduce a una simple respuesta fisiológica, a estímulos externos. Son seres que, al igual que nosotros, básicamente se interesan por evitar el sufrimiento y que pueden no conseguirlo en virtud de cómo nos comportemos con ellos. ¿Por qué no mantener entonces que la moralidad es una cuestión simplemente de no dañar a todo aquel que pueda ser dañado?». Lara (2006), p. 110.
} 
muchos animales con nosotros en aspectos relevantes para la identificación del dolor 27 .

Finalmente, la ampliación de la comunidad moral tendrá que ir seguida de una discusión en torno a cómo se concretan las obligaciones que tenemos hacia los animales y qué fuerza han de tener estas. Los deberes que supone para nosotros el reconocimiento de su considerabilidad moral es algo que necesariamente habrá que determinar.

\section{Referencias bibliográficas}

CARRUTHERS, P. (1992): The animals issue: moral theory in practice, Cambridge, Cambridge University Press.

CAVAliERI, P. (2001): The animal question. Why nonhuman animals deserve human rights, Oxford, Oxford University Press.

DAwkINS, M. S. (1986): "The scientific basis for assessing suffering in animals", en P. Singer (ed.) (2006), In defense of animals. The second wave, Oxford, Blackwell Publishing.

DeGraziA, D. (1996): Taking animals seriously. Mental life and moral status, Cambridge, Cambridge University Press.

De LoRA, P. (2003): Justicia para los animales. La ética más allá de la humanidad, Madrid, Alianza.

Dombrowski, D. A. (1997): Babies and beasts, Urbana, University of Illinois Press. FeINBERG, J. (1980): “Abortion”, en T. Regan (ed.) (1980), Matters of life and death, New introductory essays in moral philosophy, New York, Random House.

Francione, G. L. (2000): Introduction to animal rights. Your child or the dog?, Philadelphia, Temple University Press.

García Trevijano, C. (1999): "Selección histórica de textos sobre el estatuto ético de los animales", Teorema, 18.

GerreK, M. L. (2004): "Hume and our treatment of animals", Essays in Philosophy, 5. GRACIA, D. (2002): "Los animales en la tradición occidental", en J. R. Lacadena (ed.), Los derechos de los animales, Bilbao, Desclée De Brouwer.

GRUZALSKI, B. (2004): "The ability to be moral fails to show that humans are more valuable that nonhuman animals", Essays in Philosophy, 5.

Kuhse, H. y Singer, P. (1985): Should the baby live?, Oxford, Oxford University Press.

27 Para conocer los argumentos véase Singer (1975), pp. 46-49; Dawkins (1986), pp. 26-39; Mateos (2003). 
LaFollette, H. y Shanks, N. (1996): “The origin of speciesism”, Philosophy, 71.

LARA, F. D. (2006): "La entidad moral de los animales y nuestras obligaciones con ellos", Signos Filosóficos, 8.

LeAHY, M. (1991): Against liberation, London and New York, Routledge.

LEOPOLD, A. (1949): A sand county almanac with other essays on conservation, New York, Ballantine Books, (1966).

LinZEY, A. (1994): Los animales en la teología, Barcelona, Herder, (1996).

MaClean, A. (1993): The elimination of morality. Reflections on utilitarianism and bioethics, London and New York, Routledge.

Mateos, C. (2003): Bienestar animal, sufrimiento y consciencia, Cáceres, Universidad de Extremadura.

MCMahan, J. (2002): The ethics of killing: problems at the margins of life, Oxford \& New York, Oxford University Press.

Midgley, M. (1983): Animals and why they matter, Athens, University of Georgia Press.

NARVeson, J. (1979): “Animal rights”, Canadian Journal of Philosophy, 7.

Nelson, J. A. (1985): "Recent studies in animal ethics", American Philosophical Quarterly, 22.

RACHELS, J. (1990): Created from animals. The moral implications of Darwinism, Oxford, Oxford University Press.

Rawls, J. (1971): A theory of justice, Cambridge, Harvard University Press.

REgan, T. (1999): "Poniendo a las personas en su sitio", Teorema, 18.

SAlt, H. S. (1892): Animals' rights, Clarks Summit, International Society for Animal Rights, (1980).

SAPONTZIS, S. F. (1987): Morals, reason and animals, Philadelphia, Temple University Press.

SCAnlon, T. M. (1998): What we owe to each other, Cambridge, Mass., Harvard University Press.

SCHÖNFELD, M. (1992): "Who or what has moral standing", American Philosophical Quarterly, 29.

Schweitzer, A. (1929): Civilization and ethics, New York, MacMillan.

Singer, P. (1974): "Todos los animales son iguales", en P. Singer (2002), Desacralizar la vida humana. Ensayos sobre ética, Madrid, Cátedra, 2003.

Singer, P. (1975): Liberación animal, Madrid, Trotta, (1999).

Singer, P. (1979): Ética práctica, Cambridge, Cambridge University Press, (1995).

SteInBOCK, B. (1978): "Speciesism and the idea of equality", Philosophy, 53 (204).

TANNER, J. (2006): "Marginal humans, the argument from kinds and the similarity argument", Philosophy, Sociology and Psychology, 5 (1).

Warren, M. A. (1997): Moral status. Obligations to persons and other living things, New York, Oxford University Press. 
WiLson, S. D. (2005): "The species-norm account of moral status", Between the Species, 5.

Olga Campos Serena

Departamento de Filosofía I

Universidad de Granada

olgacampos@ugr.es 